\title{
INTERNATIONAL JOURNAL OF AUTOMOTIVE SCIENCE AND TECHNOLOGY
}

\section{Modelling and Simulation of Detailed Vehicle Dynamics for the Development of Innovative Powertrains}

\author{
Shantanu Pardhi ${ }^{1 *}$, Ajinkya Deshmukh ${ }^{1}$ and Hugo Ajrouche ${ }^{1}$ \\ 0000-0002-6325-2796, 0000-0002-0401-9521, 0000-0001-9414-0247
}

${ }^{1}$ Altran Prototypes Automobiles, Hybrid Innovative Powertrain, Research \& Innovation Department, ALTRAN part of Capgemini-France. 2 Rue Paul Dautier, 78140 Vélizy-Villacoublay, France

\begin{abstract}
Numerical simulation with a basic representation of longitudinal vehicle dynamics is known to be sufficient for initial powertrain development activities related to efficiency and emissions such as concept application, optimal sizing, analysing the effects of physical and functional changes and defining basic control laws. However, when it comes to comprehensive analysis for efficiency improvement, minimizing instantaneous emission peaks or studying the impact of the new concepts on road safety, drivability and performance, the significance of detailed vehicle dynamics cannot be ignored. The work presented in this article defines a forward type longitudinal vehicle dynamic modelling approach considering important characteristics such as the influence of normal load transfer on varying grip of the front and rear wheels, effect of wheel slip, and a complete representation of resistances encountered against vehicle motion with the objective of taking the analysis even closer to the actual driving conditions. The behaviour of this combined simulation platform under normal and extreme driving conditions seems to precisely follow realworld scenarios. This approach is a first step towards future analysis, optimization and controls development for improving transient powertrain aspects such as maximizing regenerative braking under heavy deceleration or optimizing road charging in P4 parallel hybrid architecture by managing wheel slip losses.
\end{abstract}

Keywords: Longitudinal vehicle dynamics simulation; Analytical modelling; Front - rear wheel slip; Trivial suspension; Vertical load transfer; Tractive forces; Powertrain analysis; Anti-lock braking system.

\author{
Research Article \\ https://doi.org/10.30939/ijastech..931066
}

$\begin{array}{ll}\text { Received } & 01.05 .2021 \\ \text { Revised } & 00.00 .2020 \\ \text { Accepted } & 27.00 .2020\end{array}$

* Corresponding author

Shantanu Pardhi

shantanu.pardhi@vub.be

Altran Prototypes Automobiles, Hybrid Innovative Powertrain, Research \& Innovation Department, ALTRAN part of Capgemini-France. 2 Rue Paul Dautier, 78140 Vélizy-Villacoublay, France

Tel: +33605706711

\section{Introduction}

With an ever-increasing application of new and innovative concepts in the powertrain domain for fulfilling the needs of fuel efficiency, reduced pollution, road safety, performance and drivability, the automotive industry is relying more and more on mathematical simulations for faster, cost-effective, and easier adaptations [1]. A rudimentary representation of vehicle dynamics has been found to be effective in the early project phase for low level powertrain simulation activities such as new concept application, assessing the working range and understanding initial control requirements under normal driving. Once this is achieved, a need for higher precision arises for not only further minimizing energy losses or finding reasons for emission peaks, but also for analysing the impact of this concept integration on other important vehicle characteristics such as road safety, drivability and performance, which are more pronounced under extreme driving conditions [2].
To simulate the detailed longitudinal vehicle dynamics, research has been done on various modelling methodologies for taking into account the effect of transient behaviour of different components on the complete powertrain. Majdoub et al. (2011) have shown control-oriented vehicle dynamics state-space representation with Kiencke's tyre slip model for speed regulation using a nonlinear controller [3]. Real time tyre friction and slip estimation technique and its integration for varying ABS control to achieve higher braking performance is given by Singh et al. (2015) [4]. The effect of steady state and transient vehicle dynamics modelling on quarter wheel model is discussed by Jansen et al. (2010) which could be used for robust ABS simulation and development [5]. Shakouri et al. (2010) have presented a vehicle dynamics modelling method with normal load transfer using balance of moments around wheel contact points [6]. James et al. (2020) have compared the results of physical and data driven models with real tests and have shown 
better outcome with the later for specific use cases [7].

The vehicle dynamics modelling technique proposed in this work fulfils simulation needs for the advanced stages of a project while still maintaining a simple and understandable level of modelling complexity. The main originality of this work includes the integration of suitable modelling methods from the literature along with an uncommon trivial suspension representation using the balance of various force moments and vehicle motion around its centre of gravity $(\mathrm{CoG})$. The precise simulation of dynamic effects and losses in wheels and other vehicle components make this method highly effective for the authors' ongoing transient analysis and development activities such as improving regenerative braking under heavy deceleration or optimizing $\mathrm{P} 4$ hybrid powertrain road charging modes in dynamic driving. Its application can be extended to even early controls architecture development and calibration for braking, traction modules, and electronic safety measures along with their validation before implementation on a real system [5]. Its scope can also cover testing of existing Electronic Control Unit strategies and functions using Model In the Loop (MIL) and Software In the Loop applications (SIL) [1].

This article is divided into several sections. Section 2 explains the selected approach with zero-dimensional modelling of the front and rear wheel dynamics including the effect of tyre slip, transmissible force and rotational inertia, front - rear normal load transfer modelling using a trivial suspension depiction and a robust representation of different tractive resistances. Section 3, shows a simplified way of representing electronic vehicle safety and drivability systems for taking into account their effect on the vehicle dynamics while avoiding their high level of functional complexity. Section 4 presents the results of the use case vehicle simulation under normal and drastic driving conditions and finally the conclusion and some future perspectives are provided in section 5 .

\section{Modelling methodology}

A modular and generic modelling approach has been used to make sure that the proposed methodology is able to simulate the working of various possible means of upcoming propulsion technologies such as electric, thermal, hybrid or even fuel cell for vehicle applications ranging from small scooters to heavy-duty trucks. This also facilitates easy integration of any vehicle architecture such as front, rear or all-wheel drive and to study the behaviour and dynamics of individual components and their impact on the functioning of the complete powertrain. For longitudinal vehicle dynamics, a dynamic forward type modelling approach based on analytical representations has been selected. It undertakes a fixed discrete-time steps simulation, instead of linear data-driven correlations running at continuous time, to fulfil the objectives of modularity, generic representation and ease of sizing while maintaining a close comparison with real-world applications [7].

The platform consists of a vehicle block for calculating the effects of tractive resistances on vehicle speed. It consists of two separate wheel blocks representing lumped front and rear wheels including the effect of slips and rotational inertias (two-wheel approach) and a vertical load transfer model for finding instantaneous normal load distribution on the front and rear during vehicle motion (fig. 1). An in-house driver block has also been integrated to obtain accelerator and brake pedal commands depending on the difference between the actual vehicle speed and the desired speed. Since the model is forward and dynamic in nature, the driver commands could also be given by real human intervention through Driver In the Loop (DIL) approach. Using these commands, the powertrain and brake blocks develop tractive and braking torque respectively. Two identical brake models are used to separately represent braking of front and rear wheels. The powertrain for this case is considered as an ideal torque source that can instantaneously give any desired torque request within its functional limits, with the idea of being able to replace it with a detailed model of an actual prime mover. Torque from the powertrain and brakes are transmitted to front and rear wheels according to the chosen vehicle architecture and depending on their dynamics transferred to the road as tractive/braking force creating a reaction on the vehicle and affecting its speed. The instantaneous vehicle speed, which depends on the wheel dynamics, then becomes angular wheel speed and is connected back to the powertrain and brakes. Thus, the model works in an action (torque) - reaction (speed) manner and can be used to precisely represent actual vehicle behaviour.

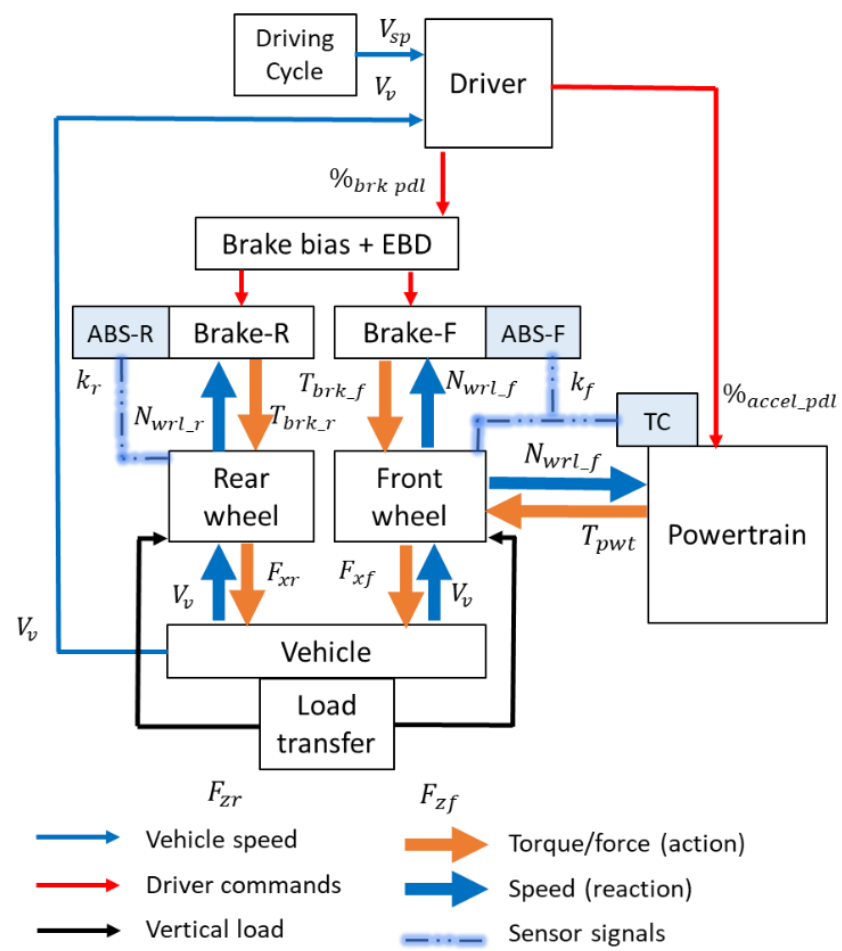

Fig. 1. Longitudinal vehicle dynamics model architecture

\subsection{Wheel model}

The wheel model calculates the amount of tractive force that the tyres transmit to the road for the vehicle to attain the desired speed and also the actual angular wheel speeds which are different from 
the vehicle speed on account of wheel slip. Under standard operating conditions (road properties) tyres have been found to follow a certain relation between the amount of force transferred to the road $F_{x}$ and their slip $k$. The amount of force transmitted also tends to be directly proportional to the normal vertical load on the wheel $F_{z}$ and the tyre friction coefficient $\mu$ (Eq. 1).

$$
F_{x}=F_{z} \mu
$$

$$
F_{z}=m_{v} g
$$

To find the amount of force that is transmitted between the road and the wheel at any moment, a widely accepted empirical correlation known as the Pacejka magic formula (MF) (fig. 2) is used (Eq. 3) which is known to represent longitudinal tyre behaviour close to the real-world application [8].

$$
\stackrel{\mu}{=} D \sin (\mathrm{C} \arctan (B k-\mathrm{E}(10 k-\arctan (B k))))
$$

The Pacjeka curve forms a correlation between the amount of tyre slip at any given moment $k$ and the corresponding transmitted force to move the vehicle $F_{x}$ [9]. It defines this correlation for both positive (tractive) and negative (braking) behaviour and can be divided into linear and non-linear operation zones. It is to be noted that the effect of tyre slip on the transmitted force is instantaneous. Linear zone correlates to the region of wheel slip where with an increase in efforts to the wheels (tractive/braking torque), higher slip is generated and greater force is transmitted to the road. For both traction and braking conditions, after surpassing a certain level of generated slip, the tyre behaviour enters the nonlinear zone where, with a further rise in slip the transmitted wheel forces tend to decrease instead of increasing, reducing the effectiveness of the tyres (fig. 2).

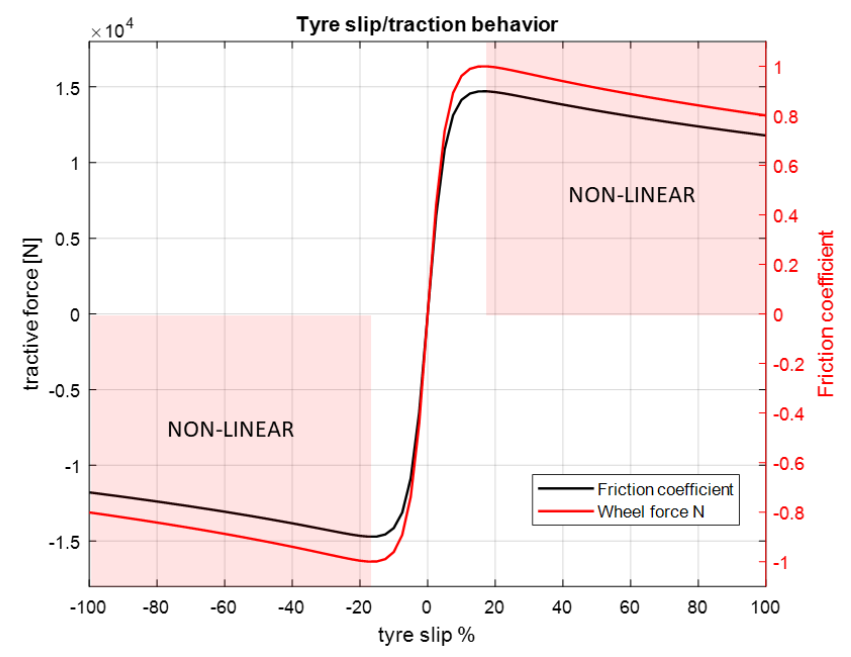

Fig. 2. Wheel slip-tractive force/Friction coefficient

Tyre slip $k$ has been calculated as the ratio of the difference between actual angular wheel speed $\omega_{w r l}$ (with slip) and ideal angular wheel speed $\omega_{\text {widl }}$ (without slip corresponding to the vehicle longitudinal speed) and the ideal angular wheel speed as seen in (Eq. 4) [9].

$$
\begin{aligned}
& k=\frac{\omega_{w r l}-\omega_{w i d l}}{\omega_{w i d l}} \\
& k=\frac{2\left(\omega_{w r l}-\omega_{w i d l}\right)}{\left(\omega_{t h}+\frac{\omega_{w i d l}{ }^{2}}{\omega_{t h}}\right)}
\end{aligned}
$$

Furthermore, slip calculation at near-zero vehicle speed is done using (Eq. 5) to avoid the error in division by zero ideal angular speed during vehicle launch or stop. A certain angular speed threshold $\omega_{t h}$ is considered for switching between the two slip formulae. The wheel torque that is actually transmitted to the road $T_{x}$, is calculated from the transmitted force $F_{x}$ described above and the wheel radius $r_{w h l}(E q .6)$.

$$
T_{x}=F_{x} r_{w h l}
$$

The remainder of torque from the powertrain $T_{p w t}$ and the mechanical brakes $T_{b r k}$ which is not transmitted to the road as $T_{x}$ (limited by wheel slip) (fig. 2), acts on the wheel's rotational inertia $J_{w h l}$ and is used to calculate the actual angular wheel speed $\omega_{w r l}$ (Eq. 7) [2]. This instantaneous remaining effort on the wheel can be positive or negative depending on the wheel dynamics (slip dependant transmitted torque $T_{x}$ ), and the torque from powertrain and mechanical braking [10].

$$
\omega_{w r l}=\frac{1}{J_{w h l}} \int\left(T_{p w t}-T_{b r k}-T_{x}\right) d t
$$

Finally, ideal angular wheel speed $\omega_{\text {widl }}$ (for zero slip) is found using the actual vehicle speed $V_{v h}$ and wheel radius $r_{w h l}$ (Eq.8). The model does not take into account tyre deformation and thus the wheel radius remains constant.

$$
\omega_{w i d l}=\frac{V_{v h}}{r_{w h l}}
$$

\subsection{Vehicle model}

A dynamic approach has been used for representing the effect of overall tractive and braking wheel force on the vehicle speed. $\boldsymbol{F}_{\text {trnsf }}$ is the force that the wheels together transmit to the road to counter aerodynamic drag resistance $\boldsymbol{F}_{\boldsymbol{a e r o}}$, rolling resistance $\boldsymbol{F}_{\text {roll }}$, longitudinal inertia $\boldsymbol{F}_{\text {inert- } \boldsymbol{l}}$ and the effect of road inclination $\boldsymbol{F}_{\text {inclin }}$ for attaining the desired vehicle speed $\boldsymbol{V}_{\boldsymbol{v} \boldsymbol{h}}$ [11] (Eq. 11). $\boldsymbol{F}_{\text {trnsf }}$ is the total force transmitted by the wheels to the road (summation of all $\boldsymbol{F}_{\boldsymbol{x}}$ wheel forces) including powertrain tractive/braking efforts and mechanical brakes efforts, $\boldsymbol{F}_{\boldsymbol{a} \text { ero }}$ depends on vehicle frontal area $\boldsymbol{A}_{\boldsymbol{v}}$, its aerodynamic drag coefficient $\boldsymbol{C}_{\boldsymbol{d}}$, air density $\boldsymbol{\rho}_{\boldsymbol{a}}$ and square of vehicle speed. 
$\boldsymbol{F}_{\text {roll }}$ depends on vehicle mass $\boldsymbol{m}_{\boldsymbol{v}}$ and rolling resistance coefficient of the tyres $\boldsymbol{C}_{\boldsymbol{r} \boldsymbol{r}}$. $\boldsymbol{F}_{\text {inert-l}}$ depends on longitudinal acceleration $\boldsymbol{a}_{\boldsymbol{x}}$ and vehicle mass, while $\boldsymbol{F}_{\text {inclin }}$ is affected by vehicle mass and road inclination $\boldsymbol{\theta}$.

$$
\begin{aligned}
& F_{\text {trnsf }}=F_{x f}+F_{x r} \\
& F_{\text {inert }-l}=F_{\text {trnsf }}-F_{\text {aero }}+F_{\text {roll }}+F_{\text {inclin }} \\
& \frac{d V_{v}}{d t} \\
& =\frac{F_{\text {trnsf }}-\frac{1}{2} \rho_{a} A_{v} C_{d} V_{v}^{2}+C_{r r} m_{v} g \cos \theta+m_{v}}{m_{v}}
\end{aligned}
$$

\subsection{Load transfer model}

The normal load transfer between the front and rear wheels has been considered using a quasi-steady state approach with respect to the pitch $\boldsymbol{p}_{\boldsymbol{y}}$ and heave $\boldsymbol{z}$ movement of the vehicle body. Depending on the actual vehicle speed, acceleration and the overall applied tractive force, the model calculates the instantaneous vertical load on the front and rear end of the vehicle [12].

When the vehicle is stationary only the effect of position of centre of gravity (CoG) $\boldsymbol{l}_{\boldsymbol{f}}, \boldsymbol{l}_{\boldsymbol{r}}, \boldsymbol{h}$ and road inclination $\boldsymbol{\theta}$ affect the weight distribution between front $\boldsymbol{F}_{\boldsymbol{z f} \mathbf{0}}$ and rear wheels $\boldsymbol{F}_{\boldsymbol{z r 0}}$ (Eq. 12, Eq. 13).

$$
\begin{aligned}
& F_{z f 0}=\frac{m_{v} g\left(l_{r} \cos (\theta)+h \sin (\theta)\right)}{l_{f}+l_{r}} \\
& F_{z r 0}=\frac{m_{v} g\left(l_{f} \cos (\theta)-h \sin (\theta)\right)}{l_{f}+l_{r}}
\end{aligned}
$$

As the vehicle moves, the deformation of front $z_{f}$ and rear $z_{r}$ suspension creates reaction forces, which vary the load transfer on the front $F_{z f}$ and rear $F_{z r}$ axles (wheels) (Eq. 14, Eq. 15). The $z_{r f}$ and $z_{r r}$ are suspension deformations due to vertical wheel movement on accounts of road unevenness.

$$
\begin{aligned}
& F_{z f}=F_{z f 0}+c_{f}\left(z_{r f}-z_{f}\right) \\
& F_{z r}=F_{z r 0}+c_{r}\left(z_{r r}-z_{r}\right)
\end{aligned}
$$

Pitch $z$ and heave $p_{y}$ movements are used to calculate the respective suspension deformations (springs + tyres + links) for the front $z_{f}$ and rear $z_{r}$ part of the suspension depending on the horizontal distance of the suspensions (wheel contacts) from the $\operatorname{CoG}\left(l_{f}\right.$ and $\left.l_{r}\right)$ using a representation of trivial suspension (Eq.
16, Eq. 17) (fig. 3).

$z_{f}=z-l_{f} p_{y}$

$$
z_{r}=z+l_{r} p_{y}
$$

These pitch and heave movements are affected by the amount of overall tractive and braking force by the wheels $F_{\text {trnsf }}$, aerodynamics drag resistance $F_{\text {aero }}$, height of the aerodynamic centre $h_{a}$, the horizontal $l_{f}, l_{r}$ and vertical position of the CoG $h$, and the overall stiffness of the front $c_{f}$ and rear $c_{r}$ suspension (springs + links + tyres) $(E q .18, E q .19)$. Horizontal and vertical balance of all forces and moments around the CoG is taken into account to calculate vehicle pitch and heave displacements.

$$
\begin{aligned}
& z=-\frac{\left(c_{f} l_{f}-c_{r} l_{r}\right)}{c_{f} c_{r}\left(l_{f}+l_{r}\right)^{2}}\left(F_{\text {trnsf }} h+F_{\text {aero }}\left(h_{a}-h\right)\right) \\
& p_{y}=-\frac{\left(c_{f}+c_{r}\right)}{c_{f} c_{r}\left(l_{f}+l_{r}\right)^{2}}\left(F_{\text {trnsf }} h+F_{\text {aero }}\left(h_{a}-h\right)\right)
\end{aligned}
$$

This instantaneous load on front $F_{z f}$ and rear $F_{z r}$ is utilised in the respective wheel models to precisely consider its effect on the available tyre grip for front and rear wheels and the amount of force that they transmit $F_{x f}$ and $F_{x r}(E q .1)$ without entering the nonlinear slipping zone (fig. 2). This approach is quasi-static (speed of vertical vehicle movement is considered constant except in the longitudinal direction) and thus cannot consider the damping dynamics coming from suspension dampers, tyres, bushings, and linkages. It does not take into account the separate effect of torque given to the wheels by the brakes (unsprung) and powertrain elements (sprung), or the effect of separate transmitted forces from the front and rear wheels on the suspension.

\section{Control}

Since the core focus of this work has been on modelling and representation of longitudinal vehicle dynamics close to real conditions, simple ways for representing the actual safety control systems for maintaining regulated vehicle functioning under normal as well as intense driving are considered. The control depictions have been made to just closely study their resulting behaviour on vehicle dynamics and not their actual functioning.

\subsection{Brake bias}

It is evident from the case vehicle's centre of gravity CoG position (Sonata 2011) (Table 1) that even when the vehicle is stationary, the weight distribution is not equally shared between the front and rear wheels. 
Table 1. Vehicle parameters

\begin{tabular}{|c|c|}
\hline Vehicle name & Hyundai Sonata 2011 \\
\hline $\begin{array}{l}\text { Vehicle mass with driver }[\mathrm{kg}] \\
\qquad\left(m_{v}\right)[21]\end{array}$ & 1542.4 \\
\hline Vehicle aero drag coefficient $\left(C_{d}\right)$ & 0.28 \\
\hline Vehicle frontal area $\left[\mathrm{m}^{2}\right]\left(A_{v}\right)$ & 2.13677 \\
\hline Aerodynamic centre height $[\mathrm{m}]\left(h_{a}\right)$ & 0.543814 \\
\hline Tyre data (front - rear size) & $205 / 65 / 16-205 / 65 / 16$ \\
\hline Wheel rotational inertia $\left[\mathrm{kgm}^{2}\right]\left(J_{w h l}\right)$ & 1.06 \\
\hline Wheel radius $[\mathrm{m}]\left(r_{w h l}\right)$ & $0.3365 / 0.3365$ \\
\hline Rolling resistance $\left(C_{r r}\right)$ & 0.012 \\
\hline $\begin{array}{l}\text { MF tyre formula tuning parameters [6] } \\
\qquad \mathrm{B} / \mathrm{C} / \mathrm{D} / \mathrm{E}\end{array}$ & $10 / 1 / 1.9 / 0.9$ \\
\hline $\begin{array}{l}\text { Centre of gravity COG position [m] } \\
\quad\left(\text { front } l_{f} / \text { rear } l_{r} / \text { height } h\right)[21]\end{array}$ & $1.106678 / 1.6889 / 0.543814$ \\
\hline $\begin{array}{l}\text { Wheel base }[\mathrm{m}] \\
\left(l_{f}+l_{r}\right)[21]\end{array}$ & 2.79654 \\
\hline Static weight distribution [21] & $60.4: 39.6$ \\
\hline $\begin{array}{c}\text { Front/rear suspension spring rate } \\
{[\mathrm{N} / \mathrm{m}]} \\
\left(c_{f} / c_{r}\right) \text { (Assumed) }\end{array}$ & $50000 / 50000$ \\
\hline $\begin{array}{l}\text { Maximum overall braking torque }[\mathrm{Nm}] \\
\text { (Assumed) }\end{array}$ & 6200 \\
\hline
\end{tabular}
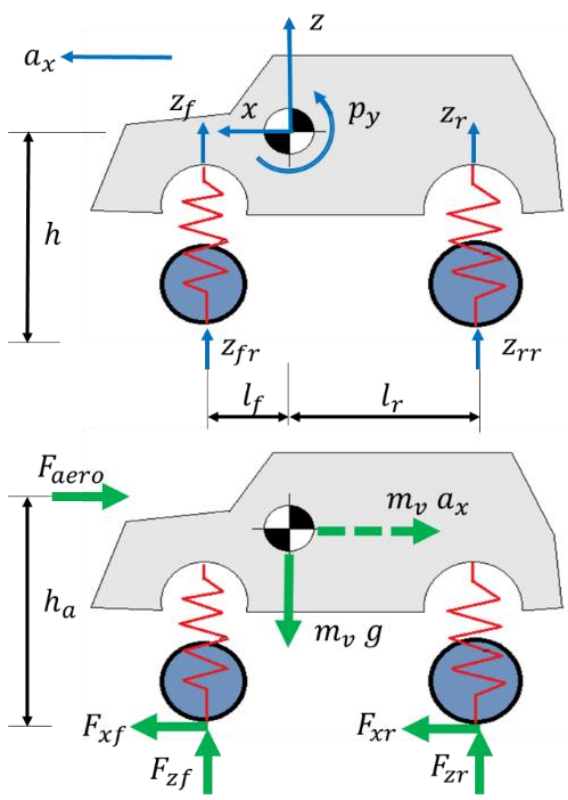

Fig. 3. Trivial suspension load transfer model

While decelerating, with the wheels stopping the vehicle and the placement of CoG pushing it forward, the vehicle tends to be thrown ahead and there is load transfer from the rear to the front suspension. This creates an even larger gap between the vertical load on the front and rear wheels and thus their ability to transmit force to the road (Eq. 5). To achieve the highest possible braking performance and road safety, this effect is taken into account and the amount of braking efforts distributed between the front and rear are altered accordingly. Brake bias is the proportion of braking efforts distributed between front and rear brakes to achieve the desired braking performance while maintaining vehicle stability [12]. In the case of a passenger vehicle, it is preferred that under heavy braking if the wheels are not able to maintain grip, it should be the front wheels that stop rotating (lockup) instead of or before the rear wheels for avoiding lateral instability and assuring occupant safety. For this application, a first calibration of brake bias has been achieved for making sure that in case of the reduced road - tyre friction (80\%) for no matter how hard the brake pedal is applied, the above safety condition is met. Due to the lack of data, the operating range of the overall braking system (braking torque) was defined so that in case of heavy braking under normal running conditions (100\% road friction), the wheel lockup limits should arise at around $80 \%$ of brake pedal input [12].

\subsection{Electronic brake force distribution}

Electronic Brake force Distribution (EBD) system or P-valve control the instantaneous brake force distribution between front and rear brake sets by decreasing braking efforts to the rear on increasing deceleration to avoid rear wheel lockup due to normal load transfer. Maintaining a less drastic brake bias under normal driving conditions helps to obtain smoother drivability and extends the life of components such as tyres and brakes. Whereas, an extreme brake distribution is useful in maintaining vehicle grip, stability and safety under heavy braking by avoiding rear lockup.

Fig. 4. Varying brake bias to avoid rear wheel lock up

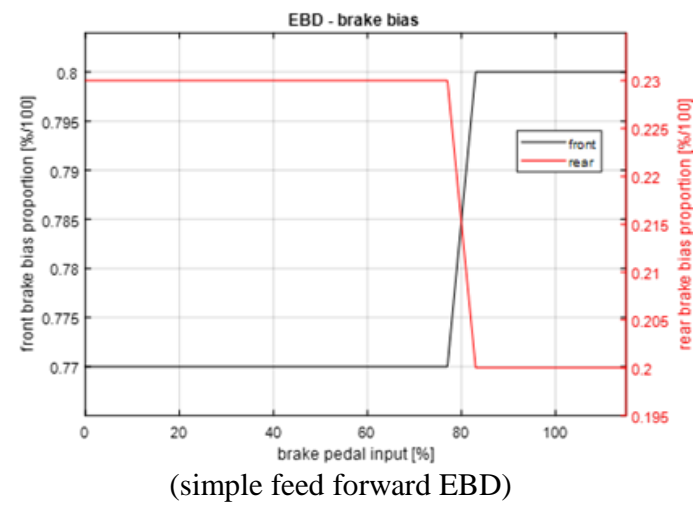

The EBD depiction for this case was a simple feedforward system which is directly based on the brake pedal input with the intention of resembling real vehicle behaviour while avoiding control system complexity. A first-level calibration for varying brake distribution was made by testing braking performance under reduced $(80 \%)$ and normal $(100 \%)$ road friction for varying brake pedal inputs to make sure that in case of a lockup the front wheels always lock instead of or before the rear wheels (fig. 4) 

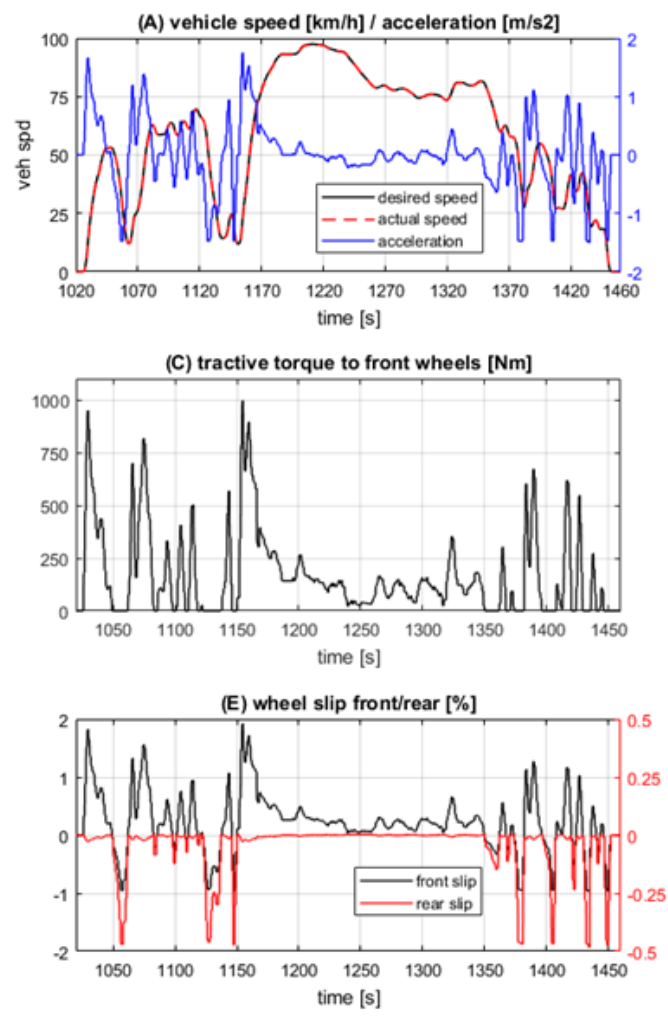

(G) pitch [deg]
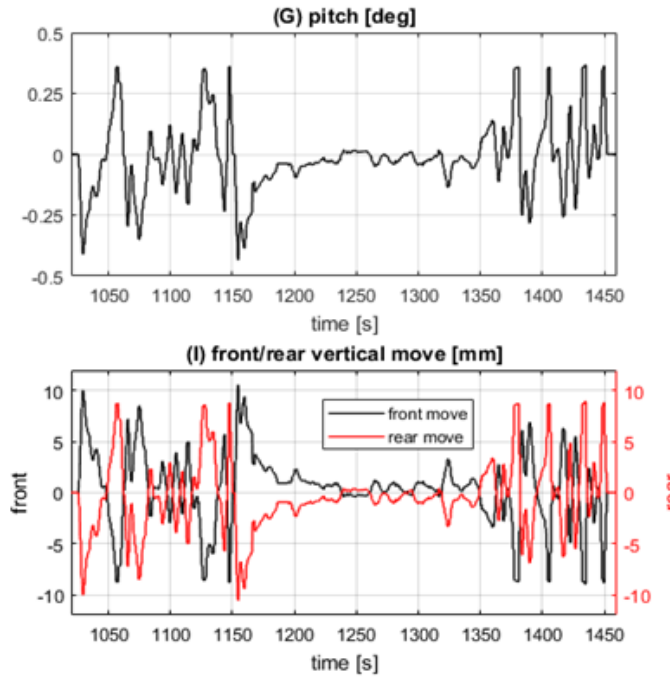
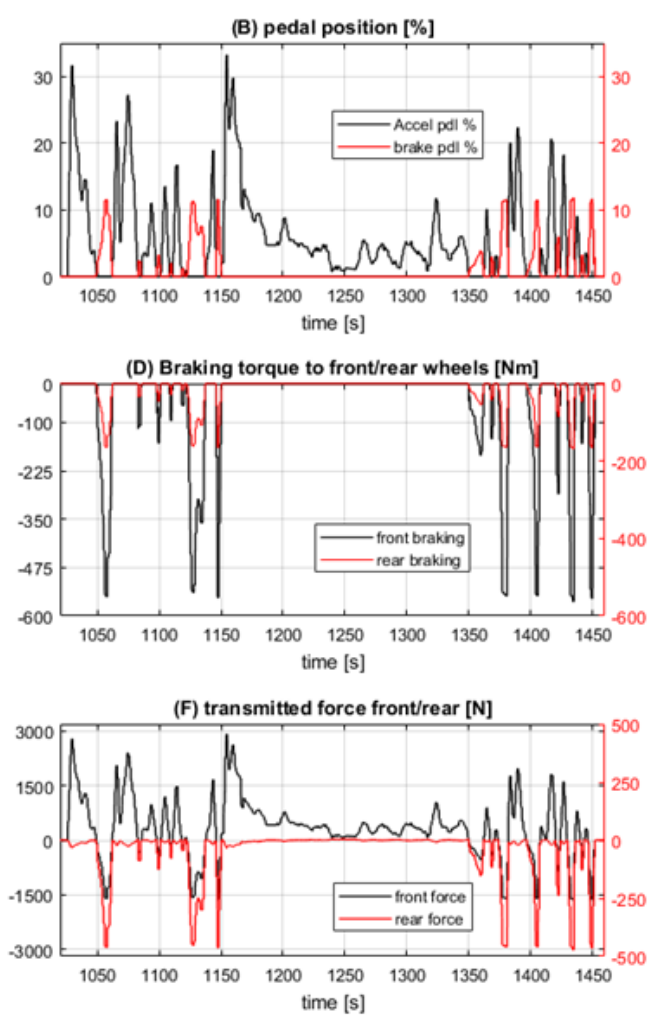

$(\mathrm{H})$ heave $[\mathrm{mm}]$
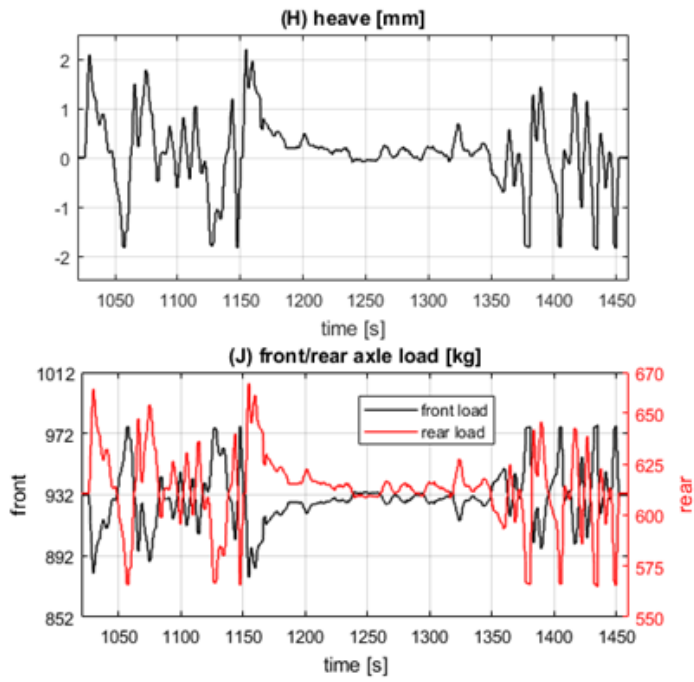

Fig. 5: Longitudinal/Vertical dynamics WLTC (1020s-1460s)

\subsection{Anti-lock braking system}

In emergency braking or during aggressive deceleration, if a high level of braking torque is applied to the wheels, they may enter the nonlinear zone of functioning after surpassing a certain level of elevated negative slip. With further increase in the wheel slip, the transmissible force to the road will start decreasing and there will be an increase in the non-transmissible braking torque remaining on the wheel which could lead to its rapid slowdown. With wheel slow down, the negative tyre slip further rises, meaning even lesser force can now be transmitted and the increasing non transmitted torque on the wheel will slow it down even further leading to its complete stop (lockup) even though the vehicle is still moving. These are uncontrollable situations where the conventional driver thinking of giving more or even 
holding braking efforts does not quickly stop the vehicle due to lesser force transmission and instead leads to a further loss of braking performance [13]. Anti-lock Braking System (ABS) maintains tyre grip during heavy braking or deceleration by avoiding high negative tyre slip $\boldsymbol{k}$ and an eventual wheel lock- up [15]. By sustaining the longitudinal grip under heavy braking, the ABS assure vehicle safety, control, performance and even better turn-ability as the lateral grip is also greatly affected by longitudinal tyre slip [16].

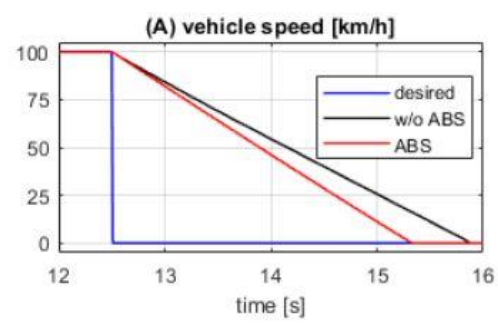

(D) deceleration [m/s2] (ABS)

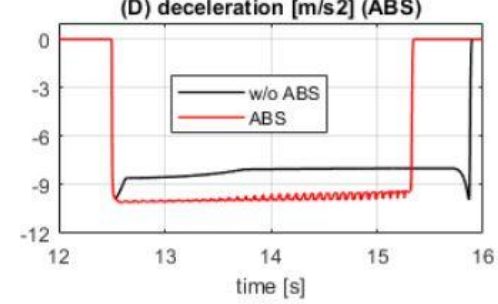

(E) Braking torque $[\mathrm{Nm}]$ (w/o $\mathrm{ABS}$ )
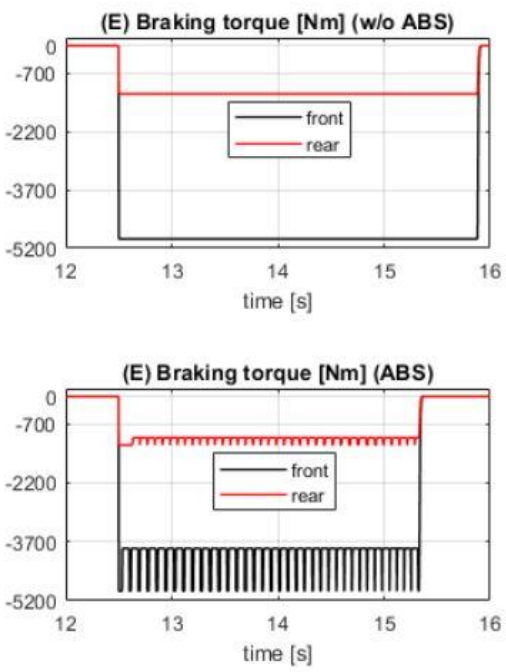

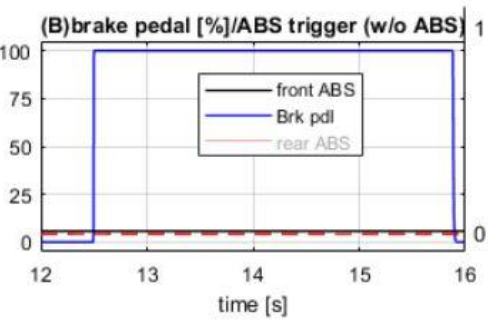

(B) brake pedal [\%]/ABS trigger (ABS)

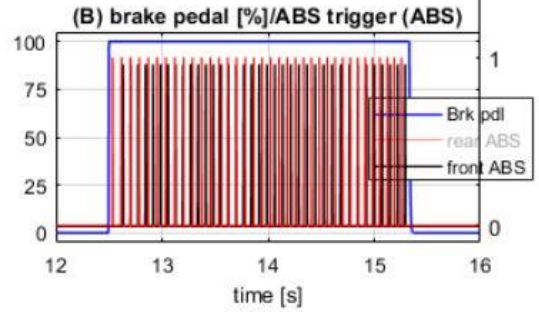

(F) Wheel slip [\%] (w/o ABS)

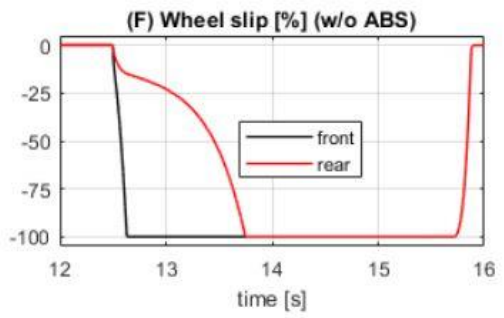

(F) Wheel slip [\%] (ABS)

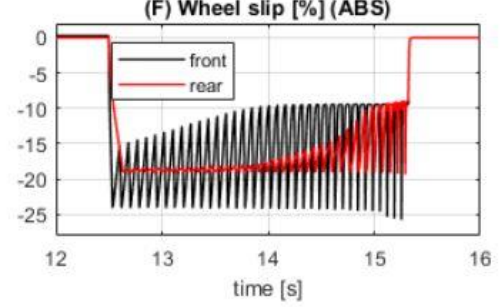

(C) Wheel speed [RPM] (w/o ABS)

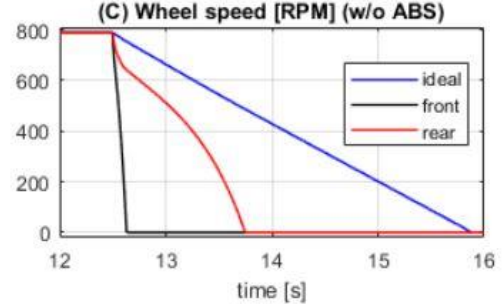

(C) Wheel speed [RPM] (ABS)

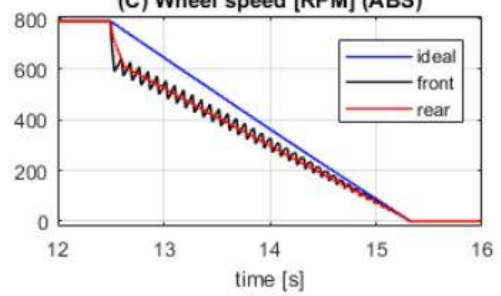

(G) Braking force [N] (w/o ABS)

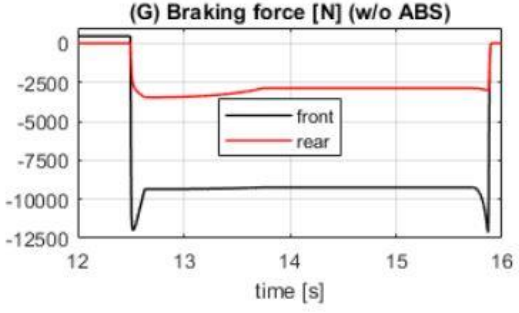

(G) Braking force $[\mathrm{N}]$ (ABS)

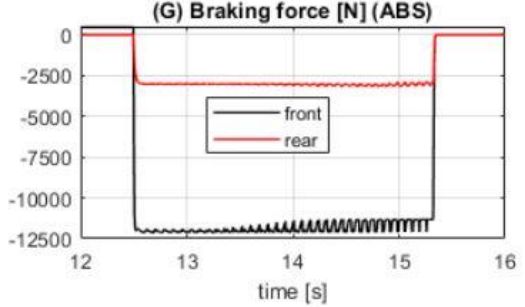

Fig. 6: Longitudinal dynamics (Braking test)

\section{Results and discussion}

\subsection{Normal driving}

The driving scenario WLTC class 3 (1020s - 1460s) has been selected to show the behaviour of the complete vehicle dynamics model with the effect of changing wheel forces, acceleration and the aerodynamic impact for the case vehicle (Sonata 2011) under normal driving (Table 1). (fig. 5) shows the important parameters related to longitudinal dynamics. The driver uses accelerator and brake pedal (fig. $5 \mathrm{~B}$ ) to make the vehicle closely follow a selected part of the driving cycle (A). Inputs from the driver are sent to the powertrain and brakes to develop tractive (C) and braking torque (D). The braking torque is directly related to the amount of brake pedal input and also to the brake bias (fig. 4) between the front and rear which leads to a difference in torque deployed to the front and rear wheels. Tractive torque is applied from the powertrain to the front wheels according to the chosen vehicle architecture (fig. 5 C). These efforts cause the slip of front and rear wheels (fig. $5 \mathrm{E}$ ). Positive tyre slip correlates to tractive force transfer between wheels and road 
for acceleration and speed maintaining while negative slip relates to braking force transmission for decelerating (fig. $5 \mathrm{~F}$ ) (fig. 2). It can be seen that the slip for rear wheels is less as compared to the front wheels during braking (D) on accounts of varying brake bias distribution. With this, the corresponding braking force transmitted is also less for the rear wheels. During acceleration or speed maintaining, it can also be seen that even if no braking torque is applied to the rear wheels there is the presence of some small amount of negative rear wheel slip (fig. $5 \mathrm{E}$ ). As the rear wheels are just supporting the weight of the vehicle, they are being dragged around (pulled) which causes some resistance due to their rotational inertia and tyre friction. This resistance to the vehicle movement can be seen as a small negative force being transmitted through rear wheels to the road $(F)$ even without the application of braking torque. In all, we see that the vehicle closely follows the desired cycle speed as the acceleration and deceleration requirements are not very drastic (A).

around CoG towards the front is considered as positive pitch and vice versa. Pitch and heave movements are directly translated in terms of deformation of the front and rear suspension with respect to their horizontal distance from the $\operatorname{CoG}(E q .16, E q .17)$. This deformation against front and rear suspension stiffness causes reactions on the wheels which equates to the amount of vertical load transferred between the front and the rear wheels (Eq. 14, Eq. 15). When accelerating the front of the vehicle rises and the rear drops (fig. $5 \mathrm{I}$ ) caused by the rotation around the
Vertical vehicle dynamics for the same part of the WLTC cycle representing normal driving are also shown in (fig. 5). As tractive force is applied via the wheels (fig. $5 \mathrm{~F}$ ), the vehicle accelerates and a part of the vertical load on the front wheels is transferred to the rear whereas when braking, it decelerates and a part of the load is transferred from the rear to the front $(\mathrm{J})$. At much higher speeds if the height of the aerodynamic centre is different from that of centre of gravity (Table 1), the aerodynamic drag force acting on the vehicle body can also lead to normal load transfer between front and rear with changing vehicle speed as the vertical distance between CoG and aerodynamic centre creates a moment of aerodynamic force acting on the CoG (lift or downforce). Thus, as the cycle advances, the vehicle speed changes, and the body is affected by moments of applied wheel force and aerodynamic force around its $\mathrm{CoG}$ which lead to body movements ( $E q$. 18, Eq. 19). Body lifting is considered positive heave and vice versa (bouncing). Body diving

CoG (pitch) (G) and also by a positive vertical displacement (heave) (H). On the other hand, when decelerating the body pitches around the $\mathrm{CoG}$ in the opposite direction towards the front and also drops in the vertical direction. This deflection of the front and rear suspension (I) is transferring the load between front and rear wheels $(\mathrm{J})$. It is to be noted that the varying of load on front and rear in $(\mathrm{J})$ is directly affecting the amount of wheel force transmitted (fig. $5 \mathrm{~F}$ ) and corresponding slip (fig. $5 \mathrm{E}$ ) for front and rear wheels.
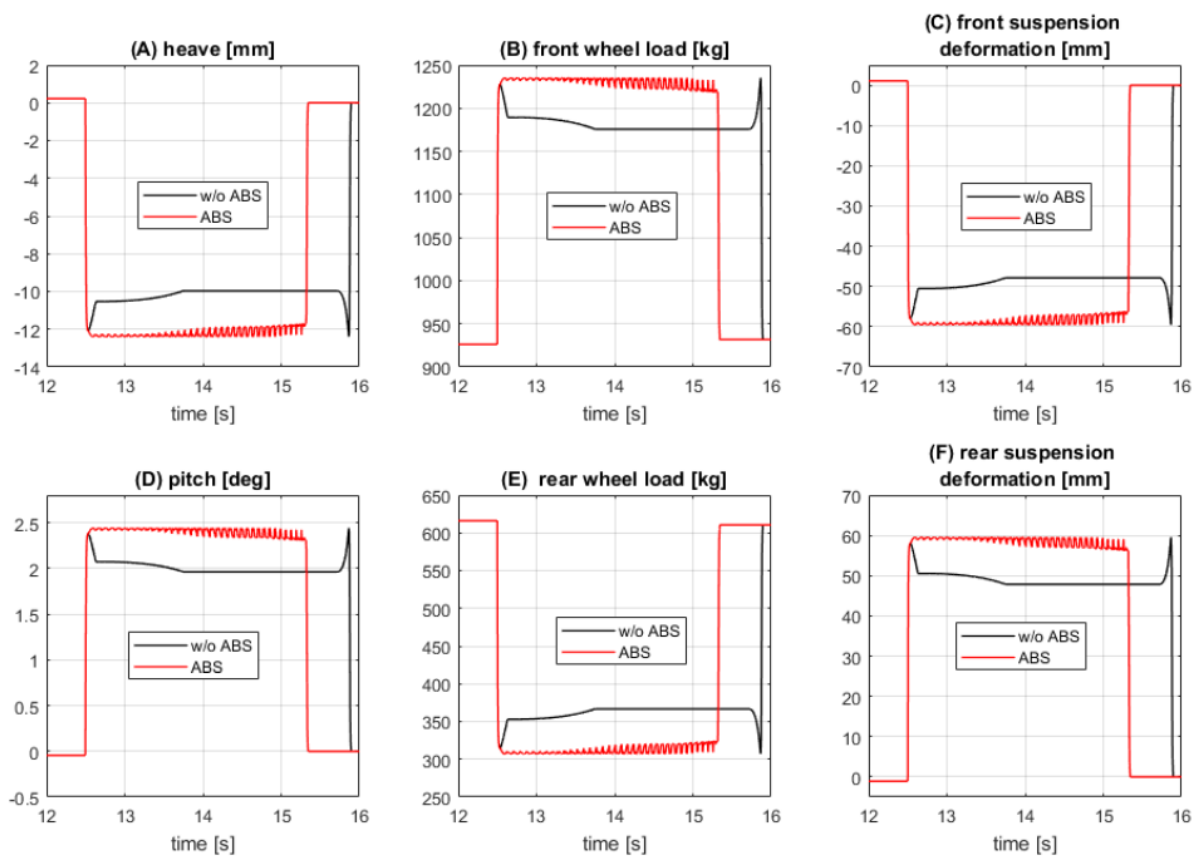

Fig. 7: Vertical Dynamics (Braking test)

\subsection{Braking test}

The wheel behaviour and the corresponding ABS operation during heavy emergency braking is shown for a $100-0 \mathrm{~km} / \mathrm{h}$ braking test. (fig. $6 \mathrm{~A}$ ) shows the desired vehicle speed set point (blue) and the decreasing actual vehicle speed during emergency deceleration. The driver brake pedal position which is the same $100 \%$ for both cases as the driver applies all of the braking ability to stop the vehicle as soon as possible (fig. $6 \mathrm{~B}$ ) according to the conventional thinking (above without ABS and below ABS). For the w/o ABS case the system is not triggered as it is absent 
while for the ABS case both front and rear control loops are triggered cyclically (black and red) with a certain delay margin (min 1/15s). (fig. 6 C) compares for w/o (above) and with ABS cases (below), the actual angular speeds for front (black), rear (red) wheel and the angular wheel speed without slip (blue) which corresponds to the longitudinal vehicle speed. As the brakes are fully applied, in absence of ABS the front wheels immediately stop rotating (lock up with -100\% slip) followed by the rear after some delay. This signifies a safe tuning of brake bias distribution under heavy braking. In the case of ABS application, the front and rear wheels continue to turn with some generated slip and are thus able to maintain higher force transmission (fig. $6 \mathrm{G}$ ) and deceleration (D) stopping the vehicle earlier (A). The Fig. (fig. $6 \mathrm{E}$ ) compares for without and with ABS application, the braking torque to the front and rear wheels. The difference in front and rear comes from the brake bias tuned for heavy braking (80:20). While the w/o ABS case continues to provide a constant braking torque to the wheels (fig. $6 \mathrm{~B}$ ), in the case of ABS as the system cycles (B), torque to both front and rear wheels are limited by some fraction (fig. 6 E) to make sure that the wheel slip is controlled (F). The system is able to control wheel slip (fig. 6 F) around the point where wheels can transmit the highest braking force (fig. $6 \mathrm{G}$ ) (fig. 2) which leads to better braking performance and shorter stopping distance (fig. 6 A). (fig. 6 F) and (C) illustrate that for the ABS case the rear wheel slip is better held and controlled much smoothly as compared to the front wheel slip, which also leads to a better control of the rear wheel braking force (fig. $6 \mathrm{G})$. This occurs as the front wheels are much weight loaded (fig. $7 \mathrm{~B}$ ) compared to the rear (fig. $7 \mathrm{E}$ ) during braking which makes them transmit higher braking force as compared to the rear (fig. $6 \mathrm{G}$ ) without entering the non-linear zone. However, as operating parameters such as rotational inertia, tyre properties (fig. 2), ABS cycling duration (1/15s), and suspension stiffness are the same for front and rear, with the application of higher braking torque to the front (fig. $6 \mathrm{E})$, the front wheel slip becomes more difficult to hold and control as compared to the rear (fig. $6 \mathrm{~F}$ ).

When comparing the simulation result of the braking test with ABS against the real-world results for the case vehicle (Sonata 2011) it is seen that the stopping distance is very close (127.6 $\mathrm{ft}$.) to the real-world test (126 ft.) [18] with a difference of $1.18 \%$. As the stopping distance is greater for simulation than for realworld it can be said that among others reasons a significant one may be overall higher tyre-road friction (MF) for the actual case $[19,20]$ in comparison to the assumption made in simulation (fig. 2)

\section{Conclusions}

This study has proposed a robust and precise zero-dimensional forward modelling approach for longitudinal vehicle dynamics with a detailed consideration of transient delays and losses including the effect of various tractive resistances and front-rear separate wheel slip and rotational inertia on the vehicle motion. The impact of normal load transfer from pitch and heave movements on the instantaneous front and rear tyre grip has also been integrated to bring the model behaviour even closer to the real-world operation.
The presented approach has also been found to precisely resemble actual vehicle dynamics under normal as well as extreme conditions which is evident from the result of the braking test. A simple representation of Anti-lock Braking System has been proposed to closely capture and match the effect of electronic safety measures on the wheel slip behaviour in drastic conditions. To make sure that the varying brake bias distribution used under normal and heavy braking matches that of the real vehicle, a lucid depiction of calibrated Electronic Brake force Distribution system has been implemented.

This work has further shown the need for improving the load transfer model to incorporate the dynamic effects coming from suspension dampers, wheels, and links. To bring the model behaviour even closer to reality, the impact of unsprung mass and that of suspension geometry with pivot points could be considered in place of trivial depiction. This would also support integrating the direct effect of separate front and rear applied wheel torque and transmitted force applications on the vehicle front-rear load transfer. The presented modelling methodology shall now be used for development of new design and control concepts that focus on maximizing regenerative and hybrid powertrain efficiency under extreme conditions such as in heavy braking or intense driving.

\section{Acknowledgment}

Altran Prototype Automobile, Research \& Innovation Department, ALTRAN part of Capgemini-France, supported this work, for the research internship under the project Hybrid Innovative Powertrain (HIP). The authors would like to thank the other members of the team Guillaume Voizard, Michel Geahel, Frédéric Guimard, Adrien Chameroy and Kamal Nouri.

\begin{tabular}{|c|c|}
\hline \multicolumn{2}{|c|}{ Nomenclature } \\
\hline$V_{v h}$ & : Actual vehicle speed $[\mathrm{m} / \mathrm{s}]$ \\
\hline$V_{s p}$ & : Desired vehicle speed $[\mathrm{m} / \mathrm{s}]$ \\
\hline \%orkpdl & : Brake pedal demand $[\%]$ \\
\hline \%accelpdl & : Accelerator pedal demand $[\%]$ \\
\hline$T_{b r k}$ & : Mechanical braking torque to the wheel $[\mathrm{Nm}]$ \\
\hline$T_{p w t}$ & : Powertrain torque to the wheel $[\mathrm{Nm}]$ \\
\hline$\omega_{\text {widl }}$ & : Ideal angular wheel speed $[\mathrm{rad} / \mathrm{s}]$ \\
\hline$\omega_{\text {wrl }}$ & : Actual angular wheel speed $[\mathrm{rad} / \mathrm{s}]$ \\
\hline$F_{\text {trnsf }}$ & : Total force transmitted from wheels to $\operatorname{road}[\mathrm{N}]$ \\
\hline$F_{z f} \& F_{z r}$ & : Vertical force on front $\&$ rear suspension $[\mathrm{N}]$ \\
\hline$F_{x f} \& F_{x r}$ & : Longitudinal force by front $\&$ rear wheels $[\mathrm{N}]$ \\
\hline$k_{f} \& k_{r}$ & : Wheel slip for front $\&$ rear wheels \\
\hline$F_{x}$ & : Force transmitted to move the vehicle $[\mathrm{N}]$ \\
\hline$F_{z}$ & : Normal vertical load on the wheels $[\mathrm{N}]$ \\
\hline$F_{\text {aero }}$ & : Aerodynamic drag resistance $[\mathrm{N}]$ \\
\hline$F_{\text {roll }}$ & : Overall rolling resistance $[\mathrm{N}]$ \\
\hline$F_{\text {inert-l }}$ & : Resistance from vehicle longitudinal inertia $[\mathrm{N}]$ \\
\hline$F_{\text {inclin }}$ & : Resistance due to road inclination $[\mathrm{N}]$ \\
\hline$A_{v}$ & : Vehicle frontal area $\left[\mathrm{m}^{2}\right]$ \\
\hline$m_{v}$ & : Vehicle mass with one passenger $[\mathrm{kg}]$ \\
\hline$Z_{r f}$ & : Front suspension deformation $[\mathrm{m}]$ \\
\hline$Z_{r r}$ & : Rear suspension deformation [m] \\
\hline
\end{tabular}




\begin{tabular}{cl}
\hline$a_{x}$ & $:$ Vehicle longitudinal acceleration $\left[\mathrm{m} / \mathrm{s}^{2}\right]$ \\
$\mu$ & $:$ Tyre friction coefficient \\
$z$ & $:$ Vehicle heave movement $[\mathrm{m}]$ \\
$P_{y}$ & $:$ Vehicle pitch angle $[\mathrm{rad}]$ \\
$C_{f} \& C_{r}$ & $:$ Overall stiffness front \& rear suspension $[\mathrm{N} / \mathrm{m}]$ \\
$h_{a}$ & $:$ Height of aerodynamic centre $[\mathrm{m}]$ \\
$C_{r r}$ & $:$ Coefficient of rolling resistance \\
$C_{d}$ & $:$ Vehicle aerodynamic drag coefficient \\
$\rho_{a}$ & $:$ Air density $\left[\mathrm{kg} / \mathrm{m}^{3}\right]$
\end{tabular}

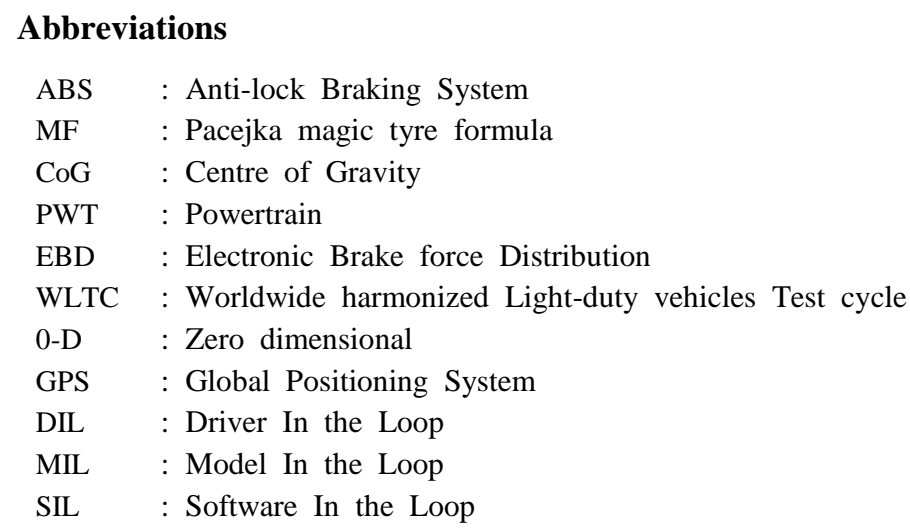

\section{Conflict of Interest Statement}

The authors declare that there is no conflict of interest in this study.

\section{CRediT Author Statement}

Shantanu Pardhi: Modelling, Simulation, validation \& writing of the original draft of the Proposed work.

Ajinkya Deshmukh: Modification \& formatting of the work. Hugo Ajrouche: Supported the proposed work.

\section{References}

[1] G. Vandia, N. Cavinaa, E. Cortia, G. Mancinia, D. Moroa, F. Pontia, \& V. Ravaglioli, Development of a software in the loop environment for automotive powertrain systems, Energy Procedia, 2014;45:789798.

[2] N. M'Sirdi, A. Rabhi, \& A Elhajjaji, Estimation of Contact Forces and Tire Road Friction, Mediterranean Conference on Control \& Automation, 2018.

[3] K. Majdoub, F. Giri, H. Ouadi, L. Dugard, \& F. Chaoui, Vehicle Longitudinal Motion Modeling for nonlinear control, Control Engineering Practice, Elsevier, 2012;20(1):69-81.

[4] K. Singh \& S. Taheri, Estimation of tire-road friction coefficient and its application in chassis control systems, Systems Science \& Control Engineering, 2015;3(1):39,61.

[5] S. Jansen, P. Zegelaar, \& H. Pacejka, The Influence of In-Plane Tyre Dynamics on ABS Braking of a Quarter Vehicle Model, Vehicle System Dynamics, 2010.

[6] P. Shakouri, A. Ordys, M. Askari, \& D. Laila, Longitudinal vehicle dynamics using Simulink/Matlab, UKACC International Conference on Control 2010.

[7] S. James, S. Anderson, \& M. Da Lio, Longitudinal Vehicle Dynamics: A Comparison of Physical and Data-Driven Models Under Large-Scale Real-World Driving Conditions, IEEE Access, 2020; 8: 73714-73729

[8] H. Pacejka \& E. Bakker, The magic formula tyre model, 1st International Colloquium on Tyre Models for Vehicle Dynamics Analysis, Delft, Netherlands, 1991.

[9] K. Lundahl, K. Berntorp, B. Olofsson, J. Aslund, \& L. Nielsen, Studying the influence of roll and pitch dynamics in optimal road-vehicle maneuvers, 23rd International Symposium on Dynamics of Vehicles on Roads and Tracks, 2013.

[10]T. Hoang, Switched observers and input-delay compensation for anti-lock brake systems, Université Paris Sud - Paris XI, 2014.

[11]L. Guzzella, \& A. Sciarretta, Vehicle Propulsion Systems: Introduction to Modeling and Optimization, Springer, 2013.

[12]B Jacobson et al, Vehicle Dynamics Compendium for course MMF062, Vehicle Dynamics Group, Division of Vehicle and Autonomous Systems, Department of Applied Mechanics, Chalmers University of Technology, 2016.

[13]S. Choi, Antilock Brake System with a Continuous Wheel Slip Control to Maximize the Braking Performance and the Ride Quality, IEEE Transactions on Control Systems Technology, 2008;16(5):996-1003.

[14]F. Sandhu, H. Selamat, \& Y. Sam, Antilock Braking System Using Dynamic Speed Estimation, Jurnal Teknologi, 2014.

[15]N. Patra, \& K. Datta, Modeling and Control of Anti-lock Braking System, Vehicle System Dynamics, 2012.

[16]T. Matsushita et al, ABS Control Unit, Fuhitsu Ten Tech. Journal, AISIN, Toyota, 1994.

[17]N. Kudarauskas, Analysis of emergency braking of a vehicle, TRANSPORT, 2007.

[18]S. Evans \& E. Lohwriter, Motortrend, [Online] 2010. https://www.motortrend.com/cars/hyundai/sonata/2011/2011hyundai-sonata-2-0t-test/.

[19]Preliminary Findings of the Effect of Tire Inflation Pressure on the Peak and Slide Coefficients of Friction, US Department of Transportation, 2002.

[20]R. Lambourn, \& A. Wesley, Comparison of motorcycle and car tyre/road friction, Transport Research Laboratory, 2010.

[21]National highway traffic safety administration laboratory test procedure for rollover stability measurement for new car assessment program (ncap), U.S. Department of Transportation, 2013. 